\title{
RACalc - a Power Distribution Reliability Tool
}

\author{
A student project towards an open source reliability software \\ for electrical distribution systems
}

\author{
J. Setréus, \\ C. J. Wallnerström, P. Hilber \\ School of Electrical Engineering \\ KTH - Royal Institute of Technology \\ Stockholm, Sweden \\ jsetreus@kth.se,cjw@kth.se, \\ hilber@kth.se
}

\author{
C. Böös \\ Vattenfall Power Consultant AB \\ Stockholm, Sweden \\ claes.boos@vattenfall.se
}

\author{
R. Göransson \\ Sweco AB \\ Stockholm, Sweden \\ richard.goransson@sweco.se
}

\begin{abstract}
One major objective of maintenance management activities in electrical distribution systems is to find the right level of investments. Within an MSc thesis project at KTH, the probabilistic reliability software RACalc has been developed to support the decision making in the distribution system maintenance planning and risk analysis. This paper describes the algorithms in RACalc and shows on present status on RADPOW, an additional reliability tool developed within the research group.
\end{abstract}

Calculations with RACalc is in this paper exemplified with a case study on an existing Swedish distribution system, were the program is used to determine the components' importance to the system reliability indices. The result show that if the failure rate can be decreased by $10 \%$ on the $21 \%$ most important components, the overall system reliability improvement is more than $7 \%$.

Keywords-component; Reliability analysis, risk management, electrical distribution systems, GUI, RADPOW, RACalc, RCAM

\section{NOMENCLATURE}

Load point indices

$\lambda_{\mathrm{LPi}} \quad$ Expected interruption rate for load point LPi [int./yr]

$\mathrm{r}_{\mathrm{LPi}} \quad$ Expected restoration time for failures in LPi [h]

$\mathrm{U}_{\mathrm{LPi}} \quad$ Expected unavailability for LPi [h/yr]

\section{System indices}

SAIFI System Average Interruption Frequency Index [int./yr,cust.] [1]

SAIDI System Average Interruption Duration Index [h/yr,cust. ] [1]

CAIDI Customer Average Interruption Duration Index [h/int. ] [1]

ASAI Average Service Availability Index [-] [1]

EENS Expected Energy Not Served [kWh/yr]

AENS Average Energy Not Served [kWh/yr,cust.]

$\mathrm{C}_{\mathrm{s}} \quad$ Customer interruption cost for DSO [SEK/yr]

\section{INTRODUCTION}

Our society has become more dependent on reliable distribution of electricity and the demand of cost efficient systems is increasing [2]. One goal for the distribution system operator (DSO) is to find a reasonable balance between the risk of customer outages and the investment costs in the electrical distribution systems (EDS). To support the necessary decision making to reach this goal, probabilistic reliability methods applied to EDSs have been a major research topic during the last decades [3]. However, to utilize the developed mathematical methods and models in the DSO's everyday work, computer software that is easy to use is crucial. The state-of-the-art reliability software is often in-house university tools, not developed to be user friendly. Moreover, hardly any software is open source, which would likely both gain the development of new reliability methods, as well as more user friendly tools for the DSO.

The intention of this paper is to present the status and latest work of developing an open source reliability software for EDS within the RCAM research group at KTH. Two programs have been developed; RADPOW and RACalc. RADPOW was first introduced in 2003 [4] and has constantly been improved with the latest methods developed within the RCAM group since then [5]. One example is the component importance indices, which is one of the major research topic within the group [6][7]. A recent performed MSc thesis project presented in [8] resulted in RACalc, which is further described and exemplified in this paper.

This paper is organized as follows: Section III gives an introduction to RADPOW and presents the current status of the program. Serving as a background for section IV, which present the basic method algorithm implemented in RACalc, with assumptions and limitations. The section also includes a short description of the different simulation routines included in RACalc. Section V describes a case study on an existing Swedish EDS performed with RACalc. Section VI summarizes the paper and discusses further improvements and possibilities of the project, towards an open source reliability tool for EDS. 


\section{RADPOW}

RADPOW is a program for Reliability Assessment of electrical Distribution POWer systems. Models of electrical power systems including attributes such as redundancy, disconnectors and breakers can be implemented in RADPOW. The tool has both modules that use analytical calculations and modules that use Monte Carlo-simulations (valuable for e.g. sensitivity analysis). Besides classical indices such as SAIFI and SAIDI, a number of component reliability importance indices have been implemented divided into three major groups:

1) Importance of the individual component's failure rate.

2) Importance of the individual component's repair time.

3) Component maintenance potential, i.e. how the system measures would be affected by an always available component (failure free).

For all of these three groups component importance is established with respect to SAIDI, SAIFI, CAIDI, ASAI, AENS and total outage cost. In total this results in 18 importance measures for every component [6][9][10].

The result from RADPOW has been validated by earlier calculations in [4][5]. Examples of possible improvement are a reliable load flow module and an improved graphical user interface (GUI).

\section{RACALC}

The reliability evaluation in RACalc is performed with a standard load-point-driven technique, where each load point (LP) is evaluated separately. For each LP a minimal cut set vector is constructed, i.e. a list of components with direct impact on the LP's ability to supply electricity to end customers. The average LP reliability of supply is then calculated based on the vector and the included component's reliability data. The results are LP indices (e.g. $\lambda_{\mathrm{LPi}}$ and $\mathrm{U}_{\mathrm{LPi}}$ ), which are used to evaluate system reliability indices, e.g. SAIFI and SAIDI.

The simulation routines Storm, Frost and 12-month are implemented in RACalc for estimating the system reliability impact of weather and time aspects. The routines scales the component reliability input parameters and calls the standard calculation module in RACalc multiple times.

The component importance simulation routine in RACalc evaluates the components' maintenance potential in the modelled EDS. The result is a component ranking which can be used as input in decision making e.g. maintenance scheduling.

\section{A. Evaluation algorithm}

Fig. 1 shows the overall workflow for the evaluation algorithm in RACalc. The following steps are included in a standard calculation:

1) Run network algorithm that deduces a minimal cut set vector $\mathrm{MC}_{\mathrm{LPi}}$ for each $\mathrm{LP}_{i}$. $\mathrm{i}=1, . .$, nno. of $\left.L P s\right\}$.

2) Run network algorithm that deduces an additional component vector with subcritical components, $\mathrm{AMC}_{\mathrm{LPi}}$, for each $\mathrm{LP}_{i}$. This vector include components not included in $\mathrm{MC}_{\mathrm{LPi}}$, but that still causes an outage in $\mathrm{LP}_{i}$ if it fails.

3) Set next $\mathrm{LP}_{i}$ to evaluate. $\mathrm{i}=1, . .,\{$ no. of $\mathrm{LPs}\}$. Summarize the total number of customers connected to $\mathrm{LP}_{i}$.

4) Set a pointer $j$ at the first element in the minimal path vector $\mathrm{CC}_{\mathrm{LPi}}$.

5) Calculate the expected failure rate, repair time and unavailability for the component at element $j$ and add this to $\mathrm{LP}_{i}$ 's total outage rate and total unavailability.

6) If pointer $j$ is not at the end of $\mathrm{CC}_{\mathrm{LPi}}$ increment $j$ and return to step 5 .

7) Set a pointer $k$ at the first element in the subcritical vector $\mathrm{SC}_{\mathrm{LPi}}$.

8) Calculate the expected failure rate, restoration time and unavailability for the component at element $k$ and add this to $\mathrm{LP}_{i}$ 's total indices. The restoration time is the time to perform corrective actions (e.g. switching disconnetors).

9) If pointer $k$ is not at the end $\mathrm{SC}_{\mathrm{LPi}}$ increment $k$ and return to step 8 .

10) Calculate the load point indices $\lambda_{\mathrm{LPi}}, \mathrm{r}_{\mathrm{LPi}}$ and $\mathrm{U}_{\mathrm{LPi}}$ for $\mathrm{LP}_{i}$.

11) Repeat from step 3 with the next LP to evaluate.

12) Calculate the system reliability indices SAIFI, SAIDI, CAIDI, ASAI, EENS, AENS and $\mathrm{C}_{\mathrm{s}}$.

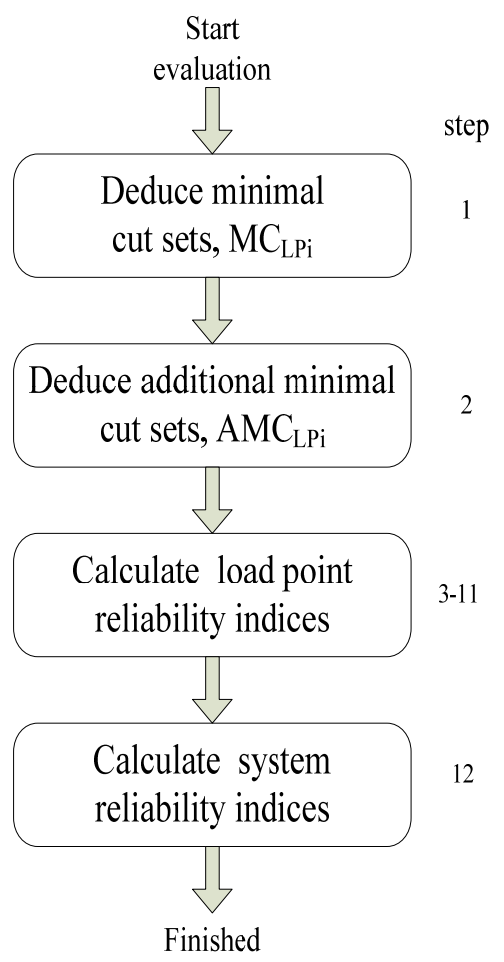

Fig. 1 General workflow for the main evaluation algorithm in RACalc. 


\section{B. Simulation routines in $R A C a l c$}

\section{1) Storm simulation}

The Storm simulation routine is designed to evaluate an approximate value of the reliability impact of a severe weather event in the studied EDS. This type of event dependency is in RACalc implemented by scaling the failure rates with estimated factors. How much a storm event increases the system reliability indices depends to some extent on the studied system and e.g. the proportion of overhead lines. Fig. 2 shows the workflow of the storm simulation routine in RACalc. First the component failure rates are scaled with user defined values and then a standard reliability calculation is performed. Table I shows the preliminary scale factors that are used in the storm simulation routine. The values were chosen to illustrate system sensitivity to certain types of components.

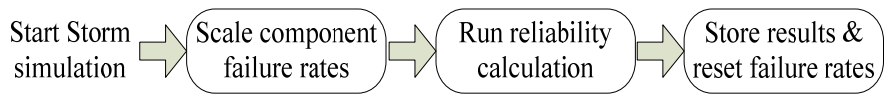

Fig. 2 RACalc workflow for storm simulation routine

TABLE I

PRELIMINARY SCALE FACTORS FOR COMPONENT FAILURE RATES IN STORM SIMULATION ROUTINE

\begin{tabular}{|l|c|}
\hline \multicolumn{1}{|c|}{ Component type } & Scale factor \\
\hline Overhead line & 10 \\
\hline Overhead cable & 3 \\
\hline All other types & 1 \\
\hline
\end{tabular}

\section{2) Frost simulation}

The frost simulation routine in RACalc is implemented to estimate the system reliability impact of an increased restoration time during a Swedish winter. Snow and frost generally increases the restoration time. Fig. 3 shows the workflow for the routine. The frost simulation scales the restoration time according to the preliminary factors in Table II. This approach assumes that the component failure rates are unaffected. The scale parameters are preliminary and have been derived by discussions with a DSO.

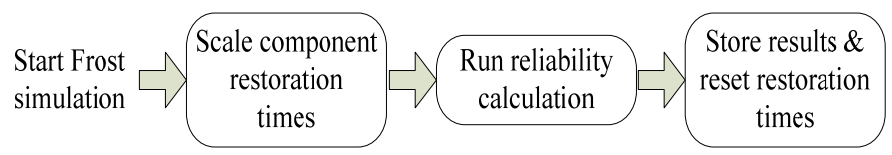

Fig. 3 Workflow of the Frost simulation routine in RACalc

TABLE II

PRELIMINARY SCALE FACTORS FOR COMPONENT RESTORATION TIME IN FROST SIMULATION ROUTINE

\begin{tabular}{|l|c|}
\hline Component type & Scale factor \\
\hline Overhead line & 1.5 \\
\hline Underground cable & 10 \\
\hline All other types & 1 \\
\hline
\end{tabular}

\section{3) 12-month simulation}

The 12-month simulation routine is designed to study the impact on the system reliability indices due to variations in component reliability data during the year. Weather and human behaviours are two reasons for these variations in component data. Fig. 4 shows the general workflow for this simulation routine in RACalc. Table III shows the failure rate scaling factors for every month during the year. This data is based on [6]. The component restoration time is assumed to be constant in this simulation routine.

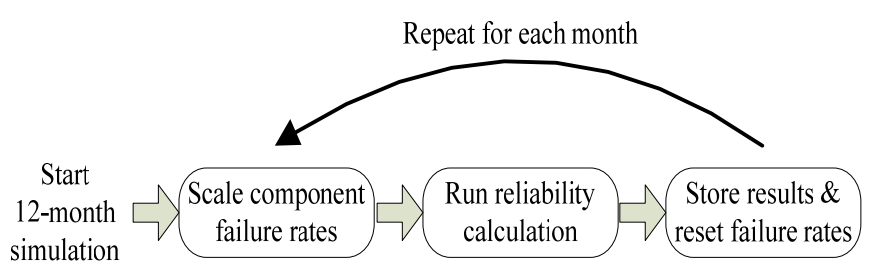

Fig. 4 RACalc workflow for the 12-month simulation routine

TABLE III

SCALE FACTORS (SF) FOR ALL COMPONENT FAILURE RATES IN THE 12-MONTH SIMULATION ROUTINE

\begin{tabular}{|cc|cc|cc|}
\hline Month & SF & Month & SF & Month & SF \\
\hline Jan & 1.11 & May & 0.83 & Sep & 0.81 \\
\hline Feb & 1.05 & June & 0.93 & Oct & 0.93 \\
\hline Mar & 1.12 & July & 1.07 & Nov & 1.02 \\
\hline Apr & 0.93 & Aug & 0.95 & Dec & 1.19 \\
\hline
\end{tabular}

\section{4) Component importance calculation}

RACalc includes a component importance index based on the maintenance potential presented in [6]. Fig. 5 shows the general workflow in the implemented simulation routine. The routine uses an iterative structure where the selected component $i$ :s failure rate is temporarily set to zero, i.e. the maximum maintenance potential. This is followed by a standard reliability calculation. The relative changes (\%) in the system indices, compared to the base case, are then stored and

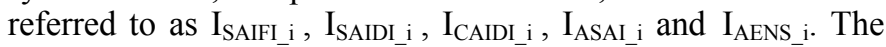
procedure is repeated for each component $i$ in the system.

An ideal component reliability improvement for maintenance is certainly not realistic, but the method approach shows on how the individual component reliability is related to the overall system reliability.

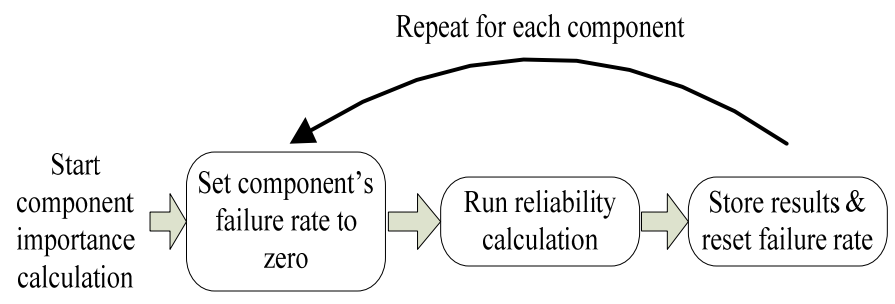

Fig. 5 Workflow of the component importance routine 


\section{Assumptions and limitations in RACalc \\ 1) Component reliability}

The component reliability model assumes an exponentially distributed restoration time and time to failure. This implies constant failure and restoration rates during the studied intervals for the components. However, during a simulation, the data parameters from one study interval (e.g. one month) to another may vary.

\section{2) Fuses}

The tripping function of fuses is currently not supported in RACalc, i.e. the component model only includes a failure rate and a restoration time. The protection function needs to be modelled and approximated with a circuit breaker in the current program version.

\section{3) Circuit breakers}

Circuit breakers are assumed to be ideal in their function of isolating faults. Hence, function faults and erratic operation is neglected in the current model.

\section{4) Redundancy}

RACalc does not currently support redundancy or meshed networks. Only radial network structures are currently supported.

\section{5) Costumer interruption cost}

The expected costumer interruption cost, $\mathrm{C}_{\mathrm{s}}$, is an estimate of the DSO's annual penalty fee for long outages in the system. In Sweden the DSOs are obligated to compensate customers suffering interruptions of supply longer than 12 hours [11]. The penalty increases with interruption time and depends on the network tariff of the customer. In the current version of RACalc all customers are assumed to have the smallest network tariff in the regulatory model.

\section{Validation of RACalc}

The reliability results from RACalc is validated in [8] by calculations of a test system. In [12] the program is validated for two test systems and one larger radial existing system, and by the use of the commercial reliability program NEPLAN [13]. The results for the two programs are similar for all cases except when fuses (tripping for e.g. resistive earth to ground faults) are introduced in the system model. This is since fuses needs to be approximated with circuit breakers in RACalc.

\section{RACALC CASE StUdy}

\section{A. Introduction}

A case study with RACalc has been performed on an EDS that is owned and operated by Sandviken Energi AB (SEEAB) in Sweden. Three subsystems of the overall EDS, referred to as ÄT34, MT8 and MT10 has been implemented in RACalc with network and statistical data received from SEEAB. The total number of components in these system models are 74 (ÄT34), 122 (MT8) and 88 (MT10).

Fig. 6 shows the original single line diagram for system ÄT34 (at top) together with the block diagram as it appear in the graphical user interface of RACalc.
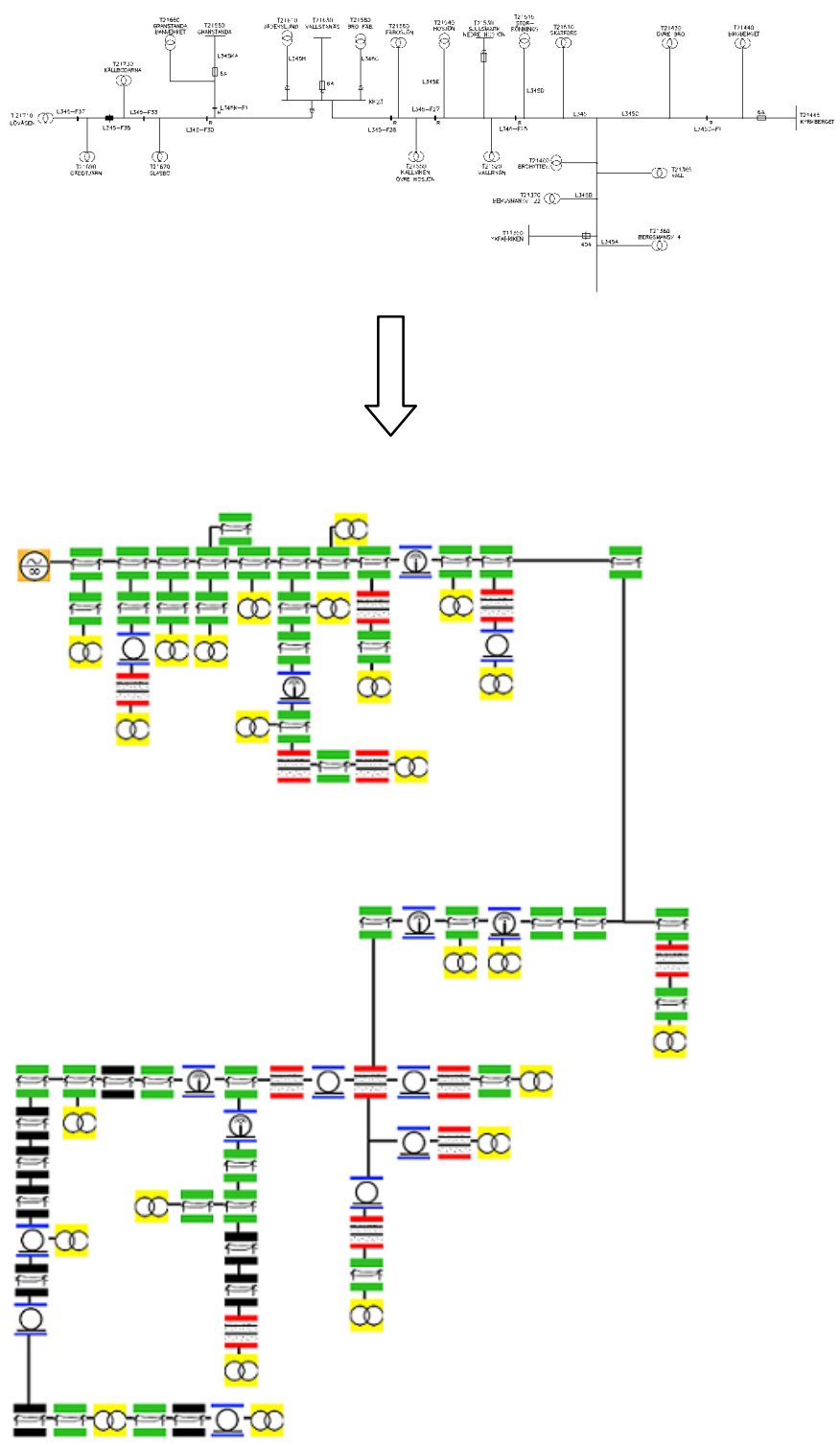

Fig. 6 Single line diagram of ÄT34 (at top) and the corrosponding block diagram model as presented in RACalc

\section{B. Case study results}

Table IV shows the system indices results from RACalc for the three studied systems. The results are realistic for the location of the studied EDS.

The component importance results from RACalc has in this case study been weighted together in order to establish one single importance metric for each component $i$. In this case study the indices $\mathrm{I}_{\mathrm{SAIFI} \_\mathrm{i}}, \mathrm{I}_{\mathrm{SAIDI} \_\mathrm{i}}, \mathrm{I}_{\mathrm{ASAI} i \mathrm{i}}$ and $\mathrm{I}_{\mathrm{AENS}_{-} \mathrm{i}}$ (improvement in \%) are summarized and form the total improvement index $\mathrm{I}_{\text {Total_i }}$ for each component $i$. In other studies the system planner may seek to improve for example 
either SAIFI or SAIDI, which will result in different component rankings.

Fig. 7-Fig. 9 illustrates the results of the improvement index $\mathrm{I}_{\text {Total_i }}$ for ÄT34, MT8 and MT10 respectively. The top twenty components for each system are shown in the figures. Fig. 10 shows the component location of the ranking (1-20) for system ÄT34. As can be seen in the figure, a large number of the topranked components are located close to the feeder node in the upper left in the model. The reason is the large number of customers that are affected downstream upon a component failure. Top ranked components that are located in the outer parts of the system (e.g. the component with rank 3), are closely connected to large customers.

TABLE IV

RELIABILITY INDICES RESULTS FROM CASE STUDY USING RACALC

\begin{tabular}{|c|c|c|c|c|c|}
\hline System & $\begin{array}{c}\text { SAIFI } \\
{[\text { int./yr] }}\end{array}$ & $\begin{array}{c}\text { SAIDI } \\
{[\mathbf{h} / \mathbf{y r}]}\end{array}$ & $\begin{array}{c}\text { CAIDI } \\
{[\mathbf{h} / \text { int.] }}\end{array}$ & $\begin{array}{c}\text { ASAI } \\
{[-]}\end{array}$ & $\begin{array}{c}\text { AENS } \\
{[\mathbf{k W h} / \mathbf{c u s t . y r} \text { ] }}\end{array}$ \\
\hline ÄT34 & 1.6 & 1.6 & 1.0 & 0.99981 & 1.5 \\
\hline MT8 & 1.4 & 2.0 & 1.5 & 0.99976 & 2.9 \\
\hline MT10 & 1.9 & 1.8 & 0.9 & 0.99979 & 1.7 \\
\hline
\end{tabular}

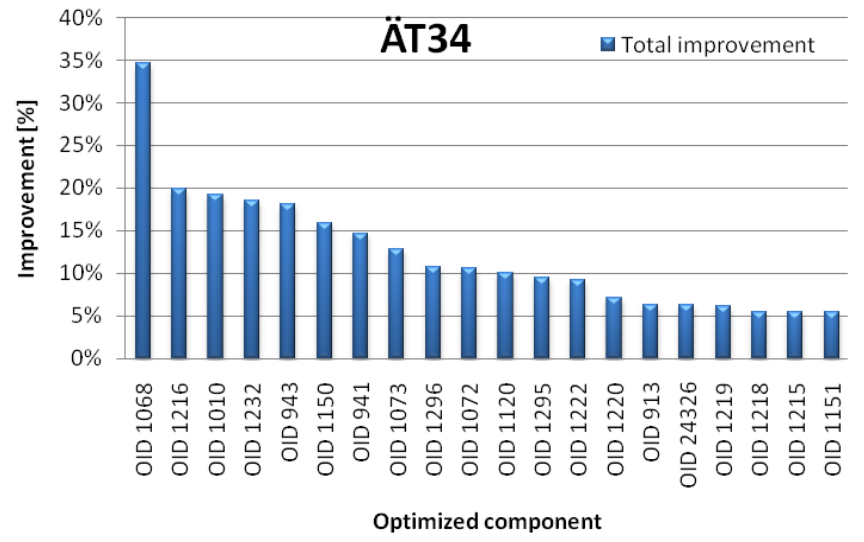

Fig. 7 The twenty highest component's total improvement index, $\mathrm{I}_{\text {Total_ } \mathrm{i}}$, for system ÄT34

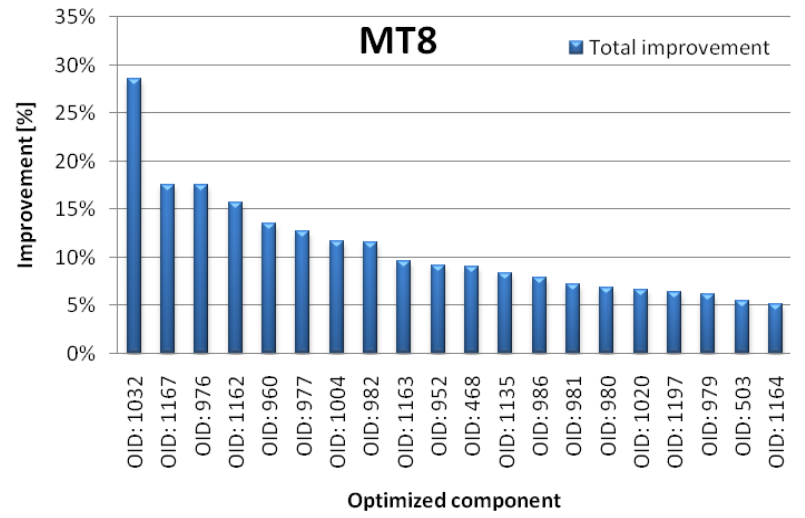

Fig. 8 The twenty highest component's total improvement index, $\mathrm{I}_{\text {Total_ } \mathrm{i}}$, for system MT8

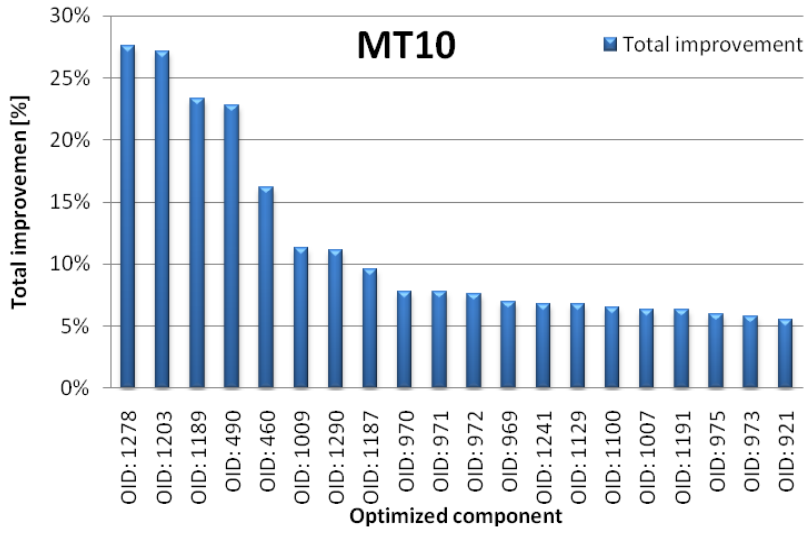

Fig. 9 The twenty highest component's total improvement index, $\mathrm{I}_{\text {Total_i }}$, for system MT10

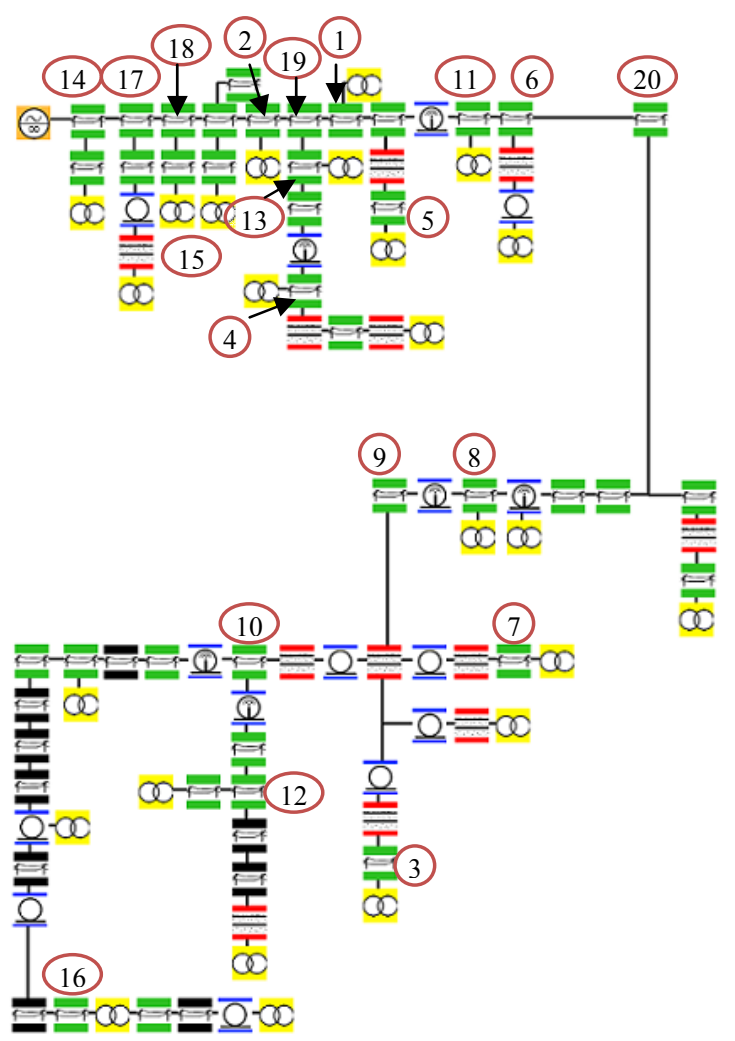

Fig. 10 The twenty highest ranked components in system ÄT34 as indicated by the component importance method in RACalc

\section{Investment scenario}

In the following investment scenario the DSO chooses to improve the twenty most important components in the systems according to the results in the previous section. In total this implies that the component reliability for $21 \%(60 / 284)$ of the total population will be improved. It is assumed that by investing more in preventive maintenance at these components (e.g. tree trimming), failure rates can be decreased with $10 \%$.

Table $\mathrm{V}$ shows the relative system indices improvement in the investment scenario for the three systems. The results show 
on an average improvement for SAIFI, SAIDI and AENS at around $7 \%$ for all three systems. The result for CAIDI, i.e. the average duration for a customer interruption, is relatively unchanged since SAIFI and SAIDI are decreased proportionally. It is interesting to note that the change for the MT8 system is on par with the other systems despite having approximately $50 \%$ more components. This indicates that further studies on how many components to be selected for improvement is crucial.

TABLE V

SYSTEM INDICES IMPROVEMENTS WHEN THE FAILURE RATE IS DECREASED BY $10 \%$ ON THE 20 MOST IMPORTANT COMPONENTS

\begin{tabular}{|c|c|c|c|c|}
\hline System & $\Delta$ SAIFI & $\Delta$ SAIDI & $\Delta$ CAIDI & $\Delta$ AENS \\
\hline ÄT34 & $8.1 \%$ & $8.3 \%$ & $0.2 \%$ & $8.2 \%$ \\
\hline MT8 & $7.3 \%$ & $7.1 \%$ & $-0.2 \%$ & $7.2 \%$ \\
\hline MT10 & $7.1 \%$ & $7.9 \%$ & $0.8 \%$ & $7.9 \%$ \\
\hline
\end{tabular}

\section{Closure}

\section{A. Conclusion}

RACalc is a tool for evaluating system reliability indices in radial distribution systems. Implemented scenario routines enable to estimate the reliability impact of weather and human behaviours in the system. This feature is especially beneficial in risk analysis including extreme outage event scenarios. Furthermore, the component reliability importance method in RACalc identifies components where preventive maintenance actions are expected to have the best system performance improvement. This information can be used as input to the DSO's risk analysis and in the decision making for more efficient maintenance management.

The conducted case study on a Swedish distribution system shows on the potential benefits of the methods and software. A total system reliability improvement of about $7 \%$ can be gained if the failure rate can be decreased by $10 \%$ on the $21 \%$ most important components.

\section{B. $\quad$ Future Developments}

One negative aspect of RACalc, in relation to RADPOW, is its inability to handle system redundancy. However, one advantage of RACalc is its user friendly graphical interface. Therefore, there are advanced plans of combining these two software's into one. The plan is to provide this program as an open source code, which for example gives researchers a better chance to develop tools useful within asset management of electrical distribution system.

\section{ACKNOWLEDGMENT}

The authors would like to express their gratitude to $\mathrm{Mr}$. Hans Reidemar and Mr. Mikael Eriksson at SEEAB for providing valuable support and input data for the case study. Furthermore, this work is sponsored by the Competence Centre in Electric Power Engineering (EKC2) and Elforsk, the Swedish National Safety Board and FIE, whom the authors are highly grateful to.

\section{REFERENCES}

[1] IEEE Standard 1366-2003, Guide for Electric Power Distribution Reliability Indices.

[2] C.J. Wallnerström, "On Risk Management of Electrical Distribution Systems and the Impact of Regulations". Licentiate Thesis, KTH Royal Institute of Technology, Stockholm, Sweden, Apr 2008.

[3] R. Billinton and R.N. Allan, Reliability Evaluation of Power Systems. 1994. ISBN 0-306-45259-6.

[4] L. Bertling, "Reliability-centred maintenance for electric power distribution systems", PhD thesis, KTH Royal Institute of Technology, Stockholm, Sweden, Aug 2002.

[5] L. Bertling, P. Hilber, J. Jensen, J. Setréus and C. J. Wallnerström, "RADPOW development and documentation", KTH Royal Institute of Technology, Stockholm, Sweden, Jan 2008.

[6] P. Hilber, "Maintenance Optimization for Power Distribution Systems", PhD thesis, KTH Royal Institute of Technology, Stockholm, Sweden, Apr 2008.

[7] J. Setréus, "On reliability methods quantifying risks to transfer capability in electric transmission systems", Licentiate Thesis, KTH Royal Institute of Technology, Stockholm, Sweden, May 2009.

[8] C. Böös and R. Göransson, "Reliability Centered Maintenance Tool The development and use of RACalc", Master of Science Thesis, KTH Royal Institute of Technology, Stockholm, Sweden, Feb 2009.

[9] P. Hilber and L. Bertling, "A Method for Extracting Reliability Importance Indices from Reliability Simulations of Electrical Networks". Proceedings 15th Power Systems Computation Conference, PSCC 2005, Liege, Belgium.

[10] P. Hilber and L. Bertling, "Monetary Importance of Component Reliability in Electrical Networks for Maintenance Optimization", In proceedings of the 8th International Conference on Probabilistic Methods Applied to Power System, PMAPS, 2004.

[11] J. Setréus, C.J. Wallnerström and L. Bertling. "A Comparative Study of Regulation Policies for Interruption of Supply in Sweden and UK". In proceedings of the International Conference on Electricity Distribution, CIRED, May 2007.

[12] C. Wilhelmsson and P. Hellestrand, "Undersökning av programvara för tillförlitlighetsanalys", Technical report (in Swedish), KTH Royal Institute of Technology, Stockholm, Sweden, May 2009.

[13] BCP Busarello + Cott + Partner AG, NEPLAN power system analysis, www.neplan.ch. 